\title{
Editorials
}

\section{Atrial fibrillation and stroke prevention:}

\author{
where we are and where we should be
}

Atrial fibrillation (AF) is the most common sustained cardiac arrhythmia. There are around 1 million people in England with diagnosed $\mathrm{AF}$ and an estimated further 425000 undiagnosed.' Given changing demographic factors and improved survival rates for comorbid cardiovascular conditions, prevalence is expected to increase significantly. By 2030, a predicted one in four middleaged adults will have developed AF. ${ }^{2} \mathrm{AF}$ is associated with a five-fold increase in stroke risk, and AF-related strokes are associated with a greater risk of mortality and longterm disability compared with other stroke subtypes. Yet this risk is largely modifiable with anticoagulation, which is proven to reduce ischaemic stroke risk by $65 \%{ }^{2}$

Given the potential benefit in terms of costs and patient outcomes, implementation of anticoagulation is a key target for the NHS In September 2017, Sir Bruce Keogh, as Medical Director of NHS England, launched the 'Size of the Prize' initiative, which highlights opportunities for cardiovascular disease prevention in each sustainability and transformation partnership (STP) area. He suggested that improvements in anticoagulation and cardiovascular disease management could prevent 14000 strokes across England over a 3-year period. It is usually possible to diagnose and treat AF without the need for specialist resources, meaning changes in service delivery must take place in primary care if these targets are to be achieved. How effectively are anticoagulation guidelines currently implemented and what barriers remain to further improvement?

\section{WHAT HAS CHANGED?}

Scoring systems are recommended to help decide which patients are likely to benefit from anticoagulation, balancing the related reduction in ischaemic stroke risk against an increased bleeding risk. The most recent National Institute for Health and Care Excellence (NICE) AF guideline recommends using the $\mathrm{CHA}_{2} \mathrm{DS}_{2}$-VASc score to predict ischaemic stroke risk, in place of the previous $\mathrm{CHADS}_{2}$ score. $^{3}$ Limitations existed with $\mathrm{CHADS}_{2}$, which was found to underestimate stroke risk in some patient groups. For example, patients who previously had an ischaemic stroke have an annual stroke risk $>8.5 \%$ without anticoagulation yet would only score 2 in $\mathrm{CHADS}_{2}$. A CHADS 2 score of 1-2 indicated 'moderate risk' of stroke, where anticoagulation should be 'considered' but

\section{"The fact that there are significant regional variations in anticoagulation prescribing, DOAC prescribing, and exception reporting suggests that patients are receiving unequal care across the NHS and opportunities for improvement remain.}

was not explicitly recommended. $\mathrm{CHA}_{2} \mathrm{DS}_{2}$ VASc incorporates additional recently identified stroke risk factors, including female sex, vascular disease, and age 65-74 years. Patients with a score of $\geq 2$ are now categorised as 'high risk' and recommended anticoagulation. $\mathrm{CHA}_{2} \mathrm{DS}_{2}-\mathrm{VASc}$ is also more reliable in identifying patients who truly have a low stroke risk (that is, a score of 0), where anticoagulation is not indicated. The change to $\mathrm{CHA}_{2} \mathrm{DS}_{2}$-VASc meant over $25 \%$ more patients were categorised as 'high risk' and nearly 300000 additional patients were prescribed anticoagulation as a result. ${ }^{4}$

The recommended stroke prevention treatment has also changed. Antiplatelet monotherapy is no longer indicated because aspirin was found to be less than half as effective at preventing strokes as warfarin and yet was associated with a similar bleeding risk. ${ }^{5}$ NICE now recommends the direct oral anticoagulants (DOACs), formerly referred to as novel oral anticoagulants (NOACs), as an alternative to vitamin $\mathrm{K}$ antagonists. ${ }^{3}$ Compared with warfarin, DOACs appear to be at least as effective for ischaemic stroke prevention and tend to cause fewer intracranial haemorrhages. ${ }^{6}$ As they do not require regular monitoring and have fewer drug interactions, DOACs provide a safe alternative to warfarin for patients where achieving a stable international normalised ratio (INR) was challenging. However, there is currently only a reversal agent for dabigatran and the DOACs remain around twice as expensive as warfarin, even factoring in monitoring costs. Increases in DOAC prescribing were related to a $60 \%$ increase in NHS anticoagulation costs between 2014 and 2015, and some clinical commissioning groups (CCGs) continue to recommend warfarin for all patients unless there is a clear contraindication. This may explain in part the significant variation in DOAC prescribing rates across CCGs, ranging from around $20 \%$ to $80 \%$ of total anticoagulation prescribed. ${ }^{7}$
DOACs will become cheaper once they are off patent by 2023 and, if further real-world studies support their efficacy compared with warfarin, there may be a move towards them being the first-line treatment for stroke prevention in all patients with AF.

\section{HOW WELL ARE ANTICOAGULATION GUIDELINES CURRENTLY IMPLEMENTED?}

The change in risk calculator, along with an ageing population, has led to a steady increase in both the absolute number and proportion of patients with AF who are eligible for anticoagulation. In this issue of the $B J G P$, Robson et al report their results from a review of anticoagulation prescribing and the factors associated with variations in practice across CCGs. ${ }^{4}$ They found a rise in AF prevalence between 2012/2013 and 2015/2016 from 1.52 to 1.71 per 100 registered adults, and the proportion of patients categorised as eligible for anticoagulation rose from around 55\% to over $80 \%$ with the change from $\mathrm{CHADS}_{2}$ to $\mathrm{CHA}_{2} \mathrm{DS}_{2}-\mathrm{VASc}$. The proportion of eligible patients prescribed anticoagulation rose steadily from $65.1 \%$ without exceptions in 2012-2013 to $77.9 \%$ without exceptions in 2015-2016. With exceptions excluded, over $85 \%$ of eligible patients were prescribed anticoagulation by the end of the study period. There was wide variation in rates of anticoagulation prescribing for high-risk patients across CCGs, ranging from $55 \%$ to $86 \%$.

Suboptimal anticoagulation prescribing across regions has been reported in other studies. A 2015 UK primary care study using GRASP-AF software, which included all 105000 people registered with 11 practices in Northern England, found $39.7 \%$ of high-risk patients were not on anticoagulation and 3\% had a stroke within 1 year. ${ }^{8}$ Antiplatelet monotherapy was used in around $25 \%$ of high-risk patients despite the NICE recommendation against this. 
The Oxford Vascular Study is community based, covering eight GP practices and 92728 patients. 9 Among this population there were 336 incident ischaemic strokes or systemic embolisms in patients with known AF across a 10-year period. Only $56(16.7 \%)$ were anticoagulated at the time of the event and anticoagulation was subtherapeutic in 34, meaning only 22 (6.5\%) were on adequate treatment.

\section{WHAT FACTORS ACCOUNT FOR SUBOPTIMAL ANTICOAGULATION?}

Exception reporting is recognised as a key factor associated with lower rates of anticoagulation prescribing. Robson et al found that the CCGs with the highest rates of anticoagulation prescribing had the lowest rates of exception reporting. ${ }^{4}$ Exceptions are justified for a range of reasons, including adverse reaction to anticoagulation, high bleeding risk, or patient choice. The HASBLED score estimates the annual risk of major bleeding in patients with AF and is intended to help identify potentially reversible bleeding risk factors. Many risk factors that contribute towards a higher $\mathrm{CHA}_{2} \mathrm{DS}_{2}$-VASc score also increase the HAS-BLED score, meaning there is a danger that patients at the highest risk of stroke will not be offered anticoagulation on the basis that their bleeding risk is too high. GPs' interpretation of a patient's relative stroke and bleeding risks may determine the information they provide each patient. The GP's personal experience of prescribing anticoagulation to previous patients may influence the way they discuss risk. Doctors who have prescribed anticoagulation to a patient who has had a subsequent haemorrhage have been shown to be $>20 \%$ less likely to prescribe anticoagulation to other patients in the next 90 days. ${ }^{10}$ This is entirely understandable as the patient who bled due to an anticoagulant will present to healthcare services but the patient who did not have a stroke will never be known. The researchers who developed $\mathrm{CHA}_{2} \mathrm{DS}_{2}$-VASC and HAS-BLED have produced an openaccess website that calculates the absolute risks of stroke and bleeding (chadsvasc. orgl, which can be helpful to facilitate shared decision making.

Some may argue that achieving 100\% anticoagulation is an unrealistic target. The fact that there are significant regional variations in anticoagulation prescribing, DOAC prescribing, and exception reporting suggests that patients are receiving unequal care across the NHS and opportunities for improvement remain. Research is needed to understand better these variations and explore the factors that influence local practice, including training, local guidelines, and service structure

\section{WHAT MIGHT CHANGE IN THE FUTURE?}

Recent guidelines have sought to simplify decision making around anticoagulation prescribing, yet this process may soon need refining as advances in technology result in more detected AF. Opportunistic screening for asymptomatic AF is already recommended, 2,3 but new technologies, such as blood pressure monitors and mobile phone apps that can detect an abnormal pulse, are making remote detection and screening ever easier. The risk of stroke and the benefits of anticoagulation in untreated, screendetected AF remain poorly understood. Currently, the burden of AF (that is, the proportion of the total time when a patient is in AF compared with sinus rhythm) is not taken into account in the anticoagulation decision process. Limited evidence suggests that patients with short runs of paroxysmal AF are less likely to have a stroke than those in permanent $A F$, but the relative risk of varying burdens of $A F$ is unclear.

Patients, clinicians, researchers, and policymakers have witnessed many advances in AF management in the past 5 years. Changes in AF risk calculation, anticoagulation treatments, and diagnostic technologies have been incorporated into national guidelines but current variation at a local level may account for a significant number of potentially preventable strokes. CCGs, and STPs, will need to invest time and resources to ensure they provide optimal stroke prevention for their populations.

\section{Nicholas R Jones,}

Academic Clinical Fellow in General Practice, Nuffield Department of Primary Care Health Sciences, University of Oxford, Oxford.

\section{FD Richard Hobbs,}

Professor and Head of Department, Nuffield Department of Primary Care Health Sciences, University of Oxford, Oxford.

\section{Clare J Taylor,}

GP and NIHR Academic Clinical Lecturer, Nuffield Department of Primary Care Health Sciences, University of Oxford, Oxford.

\section{Provenance}

Commissioned; externally peer reviewed.

\section{Competing interests}

FD Richard Hobbs and Clare J Taylor are board members of the European Primary Care Cardiovascular Society (EPCCS) and have spoken and chaired sessions at the EPCCS annual scientific meeting. EPCCS has received unrestricted educational grants from Bayer, Boehringer Ingelheim and Pfizer/BMS to fund its work.

\section{ADDRESS FOR CORRESPONDENCE}

\section{Clare J Taylor}

Nuffield Department of Primary Care Health

Sciences, University of Oxford, Radcliffe Primary Care Building, Woodstock Road, Oxford OX2 6GG, UK.

Email: clare.tayloraphc.ox.ac.uk

DOI: https://doi.org/10.3399/bjgp18X696257

\section{REFERENCES}

1. Evans T. Atrial fibrillation prevalence estimates in England: application of recent population estimates of AF in Sweden. Public Health England, 2017. https:// www.gov.uk/government/uploads/system/ uploads/attachment_data/file/644869/atrial fibrillation_AF_briefing.pdf laccessed 4 May 2018).

2. Kirchhof P, Benussi S, Kotecha D, et al. 2016 ESC Guidelines for the management of atrial fibrillation developed in collaboration with EACTS. Eur Heart J 2016; DOI: 10.1093/ eurheartj/ehw210.

3. National Institute for Health and Care Excellence. Atrial fibrillation: management. CG180. 2014. https://www.nice.org.uk/ guidance/CG180 laccessed 8 May 2018).

4. Robson J, Homer K, Ahmed Z, Antoniou S. Variation in anticoagulation for atrial fibrillation between English clinical commissioning groups: an observational study. Br J Gen Pract 2018; DOI: https://doi. org/10.3399/bjgp18X697913

5. Mant J, Hobbs FD, Fletcher K, et al. Warfarin versus aspirin for stroke prevention in an elderly community population with atrial fibrillation Ithe Birmingham Atrial Fibrillation Treatment of the Aged Study, BAFTA): a randomised controlled trial. Lancet 2007; 370(9586): 493-503

6. Ruff CT, Giugliano RP, Braunwald E, et al. Comparison of the efficacy and safety of new oral anticoagulants with warfarin in patients with atrial fibrillation: a meta-analysis of randomised trials. Lancet 2014; 383(9921): 955-962.

7. NHS England. Medicines optimisation dashboard. https://www.nhsbsa.nhs.uk/ prescription-data/medicines-optimisationdashboard (accessed 8 May 2018).

8. Shantsila E, Wolff A, Lip GY, et al. Optimising stroke prevention in patients with atrial fibrillation: application of the GRASP-AF audit tool in a UK general practice cohort. $\mathrm{Br} J$ Gen Pract 2015; DOI: https://doi.org/10.3399/ bjgp15X683113.

9. Yiin GS, Howard DP, Paul NL, et al. Agespecific incidence, outcome, cost, and projected future burden of atrial fibrillationrelated embolic vascular events: a populationbased study. Circulation 2014; DOI: 10.1161/ CIRCULATIONAHA.114.010942.

10. Choudhry NK, Anderson GM, Laupacis A, et al. Impact of adverse events on prescribing warfarin in patients with atrial fibrillation: matched pair analysis. BMJ 2006; 332(7534): 141-145. 